\title{
Low Price Equilibrium in Multi-Unit Auctions: The GSM Spectrum Auction in Germany*
}

\author{
VERONIKA GRIMM FRANK RIEDEL \\ ELMAR WOLFSTETTER \\ Institut f. Wirtschaftstheorie I, Humboldt Universität zu Berlin \\ Spandauer Str. 1, 10178 Berlin, Germany \\ E-mail: riedel/wolf@wiwi.hu-berlin.de
}

\begin{abstract}
The second-generation GSM spectrum auction in Germany is probably the most clear cut example of a low price outcome in a simultaneous ascending-bid auction. The present paper gives an account of the events, describes the auction rules and market conditions, and provides a theoretical explanation of low price equilibria in simultaneous, ascending-bid auctions. In particular it is shown that the low price equilibrium that implements the efficient allocation is the unique perfect equilibrium of that game.
\end{abstract}

KEYWORDS: multi-unit auctions, spectrum auctions, telecommunications, industrial organization, game theory.

JEL CLASSIFICATIONS: D44, D45.

\section{Introduction}

On October 28, 1999, the German regulatory authority for telecommunications (Regulierungsbehörde für Telekommunikation und Post), opened

\footnotetext{
* The authors served as consultants to prepare bidding for one bidder at the German GSM auction. Financial support was received by the Deutsche Forschungsgemeinschaft, SFB 373 ("Quantifikation und Simulation Ökonomischer Prozesse"), Humboldt-Universität zu Berlin.
} 
the auction of second-generation GSM radio frequencies in the 1.800 $\mathrm{MHz}$ range. Altogether ten nationwide paired frequencies (nine with a bandwidth of $2 \times 1$ and one with $2 \times 1.4 \mathrm{MHz}$ ) were auctioned to the four incumbent operators of mobile phone services in a simultaneous ascending-bid auction. Bidders had settled into their offices at the regulator's headquarters, and the general expectation was that bidding would go on for days. However, this was not to happen.

The first round of bidding started at 10:15 a.m.; the minimum bid was set to the nominal level of just DM 1 Million per (paired) $1 \mathrm{MHz}$ bandwidth. ${ }^{1}$ Bidder had 30 minutes to make their first bids. When the results of the first round of bidding were shown on the screen, already after 8 minutes, the press room was filled with murmurs and whistles: Mannesmann (M) had topped the minimum bid by apparently surprising jump bids in the order of DM 36.36 Million for frequencies 1 to 5, DM 40 Million on frequencies 6 to 9, and DM 56 Million on (the larger) frequency 10 (see Table 1 , where rows represent the rounds of bidding). ${ }^{2}$ In the second round, TMobil (T), a subsidiary of Deutsche Telekom, raised bids on the first five frequencies by slightly more than the minimum bid increment, and thus reduced bidding rights to five. As a result, it outbid the two smaller incumbents, Viag Interkom and E-Plus, who subsequently withdrew from the auction. In round three no bids were placed, and the auction was over, before it had gained momentum.

\begin{tabular}{ccccccccccc}
\hline \hline & & & \multicolumn{1}{c}{ Frequency \# } \\
& 1 & 2 & 3 & 4 & 5 & 6 & 7 & 8 & 9 & 10 \\
\hline 1 & 36.36 & 36.36 & 36.36 & 36.36 & 36.36 & 40.00 & 40.00 & 40.00 & 40.00 & 56.00 \\
& $\mathrm{M}$ & $\mathrm{M}$ & $\mathrm{M}$ & $\mathrm{M}$ & $\mathrm{M}$ & $\mathrm{M}$ & $\mathrm{M}$ & $\mathrm{M}$ & $\mathrm{M}$ & $\mathrm{M}$ \\
\hline 2 & 40.01 & 40.01 & 40.01 & 40.01 & 40.01 & 40.00 & 40.00 & 40.00 & 40.00 & 56.00 \\
& $\mathrm{~T}$ & $\mathrm{~T}$ & $\mathrm{~T}$ & $\mathrm{~T}$ & $\mathrm{~T}$ & $\mathrm{M}$ & $\mathrm{M}$ & $\mathrm{M}$ & $\mathrm{M}$ & $\mathrm{M}$ \\
\hline 3 & 40.01 & 40,01 & 40,01 & 40,01 & 40,01 & 40.00 & 40.00 & 40.00 & 40.00 & 56.00 \\
& $\mathrm{~T}$ & $\mathrm{~T}$ & $\mathrm{~T}$ & $\mathrm{~T}$ & $\mathrm{~T}$ & $\mathrm{M}$ & $\mathrm{M}$ & $\mathrm{M}$ & $\mathrm{M}$ & $\mathrm{M}$ \\
\hline \hline
\end{tabular}

Table 1: The GSM Spectrum Auction in Germany, October 1999. (Frequencies 1-9 were endowed with a bandwidth of $2 \times 1$, frequency 10 with $2 \times 1.4 \mathrm{MHz}$.)

\footnotetext{
${ }^{1}$ Incidentally, frequencies are paired because one is used to send and the other to receive information. To see why this is important, just listen to radio communication in a taxi cab which uses an unpaired frequency; there, only one party is able to speak, until the line is freed for the other party to respond.

${ }^{2}$ The Frankfurter Allgemeine Zeitung , 29-10-1999 wrote: "Ein Raunen ging durch den Saal ..., als das an die Wand geworfene Display das Ergebnis der ersten Bietrunde zeigt .... Wie würde T-Mobil auf die Überrumpelungsstrategie reagieren?"
} 
While this is by far the most clear cut and strong example of a low price outcome in a simultaneous ascending-bid spectrum auction, somewhat similar incidents were observed before in the US, where several such auctions took place since Congress gave the FCC a mandate to auction spectrum rights employing such a format in the year 1994. Not surprisingly, this has lead several observers of these auction to conjecture that simultaneous ascending-bid auction format may have low price equilibria or may be susceptible to collusion.

WEBER [1997] noted that if several units are up for sale in a simultaneous ascending-bid auction, "it becomes possible for a bidder to gain from strategic demand reduction, even if all of the items are currently priced below the bidder's valuations." However, due to the specific details of the auctions in the US, he believed that "in order to bring the auction to a conclusion at low prices, the bidders must negotiate with one another, and reach agreements" that may be communicated indirectly through their bidding, and then documented this kind of behavior, using data from various auctions. ${ }^{3}$ Similarly, CRAMTON AND SCHWARTZ [2000] interpreted events in several spectrum auctions in the US as collusive behavior, and KLEMPERER [2000] concluded form this experience in the US and in Germany that discouraging open or tacit collusion should be one of the two main issue of designing auction markets.

In the present paper, we show that the low price equilibrium observed in the GSM auction in Germany, is a plausible outcome of this kind of game, without invoking neither open nor tacit collusion. In particular, we show that, in a model that closely follows the rules and stylized fact of the German GSM auction, there is a low price perfect equilibrium that entails equal sharing of available frequencies among the two dominant bidders. Moreover, considering a slightly simplified specification of the GSM auction in Germany, this low price equilibrium is indeed the only perfect equilibrium of that game.

The low price equilibrium strategy involves immediate strategic demand reduction, to one half of the available objects, together with a defense of these objects up to their marginal valuations. Playing this low price equilibrium strategy does not require much sophistication, because it implements the competitive equilibrium allocation, which can be viewed as focal point in that game. Moreover, relative to the naive strategy of

\footnotetext{
${ }^{3}$ The auctions in the US are plagued by coordination problems that have to do with the fact that concrete frequencies are auctioned which are also confined to specific regional areas (see Section 2 below). This design makes bidding formidably difficult.
} 
truthful bidding, the equilibrium is evolutionary stable, in the sense that if bidders either bid truthfully of play the equilibrium strategy, those who play equilibrium are never worse off, and strictly better off when they meet others who also play equilibrium. At the same time, reducing bidding rights to the number of objects that one is awarded in the competitive allocation is a strong signal to the other player to follow suit.

In our analysis, the uniqueness of the low price equilibrium has a lot to do with the activity rule that is used in simultaneous ascending-bid auctions to keep bidders from laying in wait, until they see the intentions of others (see MCAFEE AND MCMiLlan [1996] and MiLgrom [1998]). ${ }^{4}$ This rule stipulates that, once a bidder has reduced his demand to a certain level, he cannot raise it thereafter. Interestingly, this rule can be used by bidders as a commitment device, which supports an early end of the auction. In this sense, the activity rule does indeed speed up the auction - more than the auctioneer desires.

There are several other contributions that deal with low price equilibria in related though different models of multi-unit auctions. Most of them concern "share auctions" in which bidders bid for shares of a continuously divisible good. Share auctions were introduced by WiLSON [1979] who already noticed, in a sealed-bid framework, that they may have low price equilibria (see also BACK AND ZENDER [1993]).

More recently, MENEzES [1996] studied an ascending-bid share auction under complete information, and established the existence of low price equilibria. Unlike in our model, the equilibrium allocation of the MENEZES [1996] model is not unique. This seems to be due to the combination of the continuous divisibility of the good with a discontinuously increasing price clock. MENEZES [1996] then employed Pareto dominance to exclude high price equilibria. Essentially, Pareto dominance rules out threat-strategies that may induce outcomes that are profitable for some bidder, yet Pareto inferior. By contrast, our analysis shows that with finitely many goods such a refinement is superfluous, because the threat strategies that MENEZES [1996] excludes by assumption cannot be part of a subgame perfect equilibrium in any case.

Similar to the model by MeNEzes [1996], AusubEL AND SCHWARTZ [1999] analyzed a share auction under complete information, specializing to two bidders and flat marginal valuations. In addition, they changed the auction rules in such a way that bidders are required to place their bids

\footnotetext{
${ }^{4}$ It has also been suggested that the activity rule is meant to prevent collusion, because it limits the means of retaliation to a breach of collusive agreements.
} 
in a given sequential order. The latter makes the auction equivalent to a sequential bargaining problem, and it is therefore not surprising that the game has a unique subgame perfect low price equilibrium, which corresponds to the immediate agreement property of the subgame perfect equilibrium of the RUBINSTEIN [1982] model of sequential bargaining under complete information.

Finally, ENGELBRECHT-WIGGANS AND KAHN [1998] introduced a model of a simultaneous ascending-bid auction with two objects and two players. They showed that there exist equilibria in which low prices result with positive probability, while high price outcomes also occur with positive probability. The advantage of their model is that it permits incomplete information; however, the limitation is the restriction to two objects and two bidders.

The plan of the paper is as follows. In Section 2 we present some stylized facts about the mobile phone market in Germany, which motivates some of our assumptions. In Section 3, we present a model of simultaneous ascending-bid multi-unit auctions, which fits very closely the actual GSM auction in Germany. We then show, in Section 4, that this auction game has a subgame perfect equilibrium in which the two dominant firms share the available licenses equally, at minimal prices. We call this equilibrium "low price equilibrium". Introducing one further simplification, we further sharpen this result in Section 5, where we show that the low price equilibrium is indeed the unique perfect equilibrium of that game. The paper ends with some conclusions in Section 6.

\section{Stylized Facts}

In mobile communications, the scarce resource is the spectrum required for radio transmission between users and base stations. The first generation analogue systems used the spectrum inefficiently, so that only few customers could be served and few providers could be licensed. A fundamental improvement in the utilization of the radio spectrum occurred with the transition from analogue to digital technology, and the introduction of the European GSM (Global System for Mobiles) standard. This technology used the spectrum much more efficiently, and it was able to accommodate four to five times as many customers. Thus, a mass market of mobile communications could develop. 
In Germany, the mobile phone industry started with commercial services in 1992 as a duopoly, served by T-Mobil (a subsidiary of the former state monopoly, Deutsche Telekom), and Mannesmann Mobilfunk. Even though they were licensed already in December 1989, they did not provide commercial service prior to mid $1992 .{ }^{5}$ When the second generation GSM 1.800 technology became available, the regulator used this opportunity to license two additional providers: E-Plus, in 1994, and Viag Interkom, in 1998. In order to give them a kick start, the regulator endowed them quite generously with second generation GSM frequencies that operate in the $1.800 \mathrm{MHz}$ range. Indeed, while Mannesmann and T-Mobil had to live with $2 \times 12,5 \mathrm{MHz}$ in the $900 \mathrm{MHz}$ spectrum each, both E-Plus and Viag were endowed with $2 \times 22,5 \mathrm{MHz}$ in the $1.800 \mathrm{MHz}$ spectrum. Nevertheless, the two early entrants became dominant firms, with almost equal market shares (each roughly $40 \%$ ), whereas E-plus (14,9\%), and in particular Viag Interkom (5,3\%), remained remarkably insignificant. ${ }^{6}$

By the time of the GSM auction in 1999, when more second-generation GSM 1.800 spectrum (previously used by the military) became available, the two dominant providers were subject to severe spectrum capacity constraints, which inhibited their growth, whereas the two latecomers were sitting on idle capacity, except in a few urban areas. Altogether, the two dominant firms were eager to finally gain access to the GSM 1.800 technology, whereas the small providers had only a limited interest in these frequencies because they could remove the bottlenecks by building additional radio stations in those urban centers, instead of buying more nationwide frequencies and upgrade existing radio stations. Moreover, it was already known that third-generation (UMTS) frequencies would be auctioned in the following year 2000.

On this background, the two dominant firms had a pretty good idea of each others' valuations for the new frequencies. Moreover, they could easily assess the valuations of the two small providers, by computing the cost of building a more closely knit network of radio stations in urban

\footnotetext{
${ }^{5}$ This long delay was primarily due to bureaucratic delays in the European wide licensing of mobile telephones.

${ }^{6}$ Incidentally, one could argue that the second generation GSM technology unduly burdened the new entrants with high capital cost. Operating in the higher 1.800 $\mathrm{MHz}$ range requires a closer network of base stations, even at low levels of capacity utilization. This combination is not the most attractive for a new entrant with a small customer base. However, this slight handicap was more than compensated by the fact that late entrants were subject to dramatically lower investment costs (roughly one half of what it was in the early 1990's) and that they benefitted from an already mature technology.
} 
centers, which was an obvious alternative to buying more nationwide frequencies.

Based on this assessment, the two dominant firms could figure out, with a reasonable degree of certainty, at which critical bid level the two smaller providers would quit the auction. So they had to decide whether it was worthwhile to predate the smaller providers, by raising the price to that critical level, or to accommodate and share the frequencies with the smaller providers, at a lower price. Given the extreme asymmetry of the endowments with GSM 1.800 spectrum at the time of the auction the dominant firms did not have any, while the latecomers had had more than the auction made available altogether - it was clear that it would not take much to predate, and therefore the right decision was obvious.

Mannesmann started with a jump bid on all frequencies (see Table 1) for two reasons: to bring the price uniformly to the critical level at which the smaller providers would quit the auction, to coordinate efficiently with T-Mobil on how to divide the frequencies numbered from 1 to 10 , and to signal the strategy to be played among T-Mobil and Mannesmann, after the exit of the two small providers. And indeed, this strategy worked smoothly. While the two small providers still made a last-minute bid on some of the first five frequencies, they did not acquire a high bid, and quit immediately thereafter. ${ }^{7}$

In the following we analyze the subsequent game played between the two dominant firms, T-Mobil and Mannesmann Mobilfunk, after the initial jump bid that succeeded to kick out the two smaller players.

\section{A Model of the GSM Spectrum Auction}

We now present a model of the GSM auction in Germany which ignores some less important aspects of the actual auction. This model is further simplified when we address uniqueness of perfect equilibrium in Section 5 .

At the outset, we mention that in the GSM auction in Germany all frequencies were nationwide and "abstract". The latter means that the regulator

\footnotetext{
${ }^{7}$ The Frankfurter Allgemeine Zeitung, 29-10-1999, reports: "Die Uhr sprang auf die letzte Minute, als der Computer noch Gebote von E-Plus ... registriert." (The clock approached the last minute when the computer registered bids by E-Plus ....) They could have bid the same as T-Mobil, since the rules of the auction prescribed that in case of a tie, the earlier bidder is the high bidder.
} 
assigned the concrete location in the spectrum after the auction. At the time of the auction, all frequencies were identical (except that one frequency had a higher bandwidth). The introduction of abstract frequencies avoids the coordination problems that plagued spectrum auctions in the US. It is a very useful innovation. ${ }^{8}$ Indeed, the regulator managed to assign concrete frequencies without much bureaucratic delay, and without much controversy. ${ }^{9}$

1. An even number of $2 n, n \in \mathbb{N}$, units of a homogeneous good (such as paired frequencies of a certain $\mathrm{MHz}$ endowment) are for sale in a simultaneous, ascending price auction (the rules of which are assumed to be known).

2. Bidders are free to bid on up to $2 n$ units; however, once a bidder has bid on $k<2 n$ units, he cannot later bid on more than $k$ units (activity rule).

3. Bids are required to be on an equidistant finite grid $G:=\{\Delta T \mid T=$ $0, \ldots, S\}$, where $\Delta$ is the minimum bid increment.

4. After each round, the auctioneer announces all previous bids.

5. There are two bidders, $M$ and $T$; these are risk neutral, and have identical valuations, that are common knowledge among them. Their marginal valuations, $v(k)$, are strict monotone decreasing in the number of units $k$.

Evidently, if a bidder is awarded $k$ units at prices $\left(p_{i}\right)_{i=1, \ldots, k}$, his payoff is $w_{k}-\sum_{i=1}^{k} p_{i}$, where $w_{k}:=\sum_{i=1}^{k} v(i)$, denotes his absolute willingness to pay for $k$ units.

For later use, we define

$$
T_{k}:=\min \{t \in\{0, \ldots, S\} \mid \Delta t \geq v(k)\} .
$$

\footnotetext{
${ }^{8}$ Incidentally, the auction was designed by Wissenschaftliches Institut für Кommunikation (WIK).

${ }^{9}$ Apparently, this does not extend to the German UMTS auction which took place in the summer of the year 2000. The main reason is that the frequencies are not the same, especially due to interference problems in the many border areas of Germany, and the fact that pan European providers have a strong preference for particular frequencies. On this background and the fact that regulator and license holders could not yet reach an agreement - almost one year after the auction - it is clear that the auctioning of abstract UMTS frequencies only postponed the problems of assigning concrete frequencies.
} 
$\Delta T_{k}$ denotes the smallest grid value above the marginal valuation $v(k)$.

Altogether, this game is a finite multistage game of complete information with observable actions. The finiteness of the game is due to the finiteness of the grid of feasible of bids, and the fact that bids must increase in each round until the auction ends. This entails that the game must end after at most $2 n(S+1)$ rounds. The complications induced by an infinite horizon game are thus avoided; for example, this rules out the (noncredible) threat of bidding on all packages forever.

This model abstracts from a few details of the actual GSM auction in Germany: 1) In the GSM auction one $2 \times 1.4 \mathrm{MHz}$ and nine $2 \times 1 \mathrm{MHz}$ abstract packages were for sale; hence, the units were not entirely homogeneous. 2) In the actual auction only high bids, and not all bids, were published; hence, the game was not exactly one with observable actions. 3) The minimum bid increment was fixed as percentage, and the auctioneer had some discretion in reducing the minimum bid increment in the course of the auction. 4) There were four players. However, as we have already explained, the two other players (Viag and E-Plus) were sitting on plenty of capacity, and it was understood that they would drop out of the auction at a fairly low price, which they did. Therefore, our analysis moves right away to the relevant game played between the two dominant firms, after the two insignificant bidders drop out of the auction.

\section{Low Price Equilibrium}

Since the two players have the same preferences, and since their marginal valuations are decreasing, the efficient allocation assigns five frequencies to each of them. It seems to be a kind of folk theorem that, in a simultaneous ascending auction, bidders may be able to coordinate on a low price equilibrium. We now prove that indeed the particular low price equilibrium which implements the efficient allocation is a subgame perfect equilibrium (SPE) outcome.

THEOREM 1 (LOW PRICE EQUILIBRIUM) The following strategies are a subgame perfect equilibrium; they apply to both $M$ and $T$, unless stated otherwise:

1. start with five bids, each at the level of 0 , on the frequencies $1, \ldots, 5$ $(T)$ and $6, \ldots, 10(M)$; 
2. don't overbid if five or more high bids are reached or if overbidding the rival's lowest high bid makes the contested frequency more expensive than valuable;

3. if less than five high bids are reached, overbid the rivals' lowest high bids as long as overbidding keeps the price of these contested below their value.

PROOF In a multistage game with observed actions a strategy profile is subgame perfect if and only if its satisfies the "one-stage-deviation condition" that no player can gain by unilaterally deviating from it in a single stage, while conforming to it thereafter. We show that the strategy profile satisfies this condition in all subgames, on and off the equilibrium path.

Consider subgames where bids have been made on all frequencies, all bids are below $v(5)$, and one bidder has five or more high bids.

Suppose $M$ has five or more high bids. Instead of refraining from bidding, let $M$ engage in the following one-stage deviation from the candidate equilibrium strategy: outbid some of $T^{\prime} s$ high bids, and then return to the equilibrium strategy. $T^{\prime} s$ response is to reclaim the lost frequencies by outbidding $M^{\prime} s$ lowest high bids. As a result, the game is back, after one stage, to where is was before the deviation, except that some of $M^{\prime} s$ high bids are at a higher level. Therefore, $M^{\prime} s$ one-stage deviation did not pay.

Next, suppose $M$ has less than five high bids. Instead of attacking $T$ in order to claim the "missing" frequencies, let $M$ engage in the following one-stage deviation from the candidate equilibrium: fight for less than five frequencies, and then return to the equilibrium strategy. After $M^{\prime} s$ one-stage deviation, $T$ has more than five high bids, and hence refrains from bidding. Consequently, the auction ends ( $M$ cannot attack $T$ any more because of the activity rule) and $M^{\prime} s$ one-stage deviation did not pay.

Other deviations, such as outbidding one's own high bids, are obviously not profitable.

Similar reasoning applies to all other subgames.

Thus, there is a subgame perfect equilibrium of the auction which generates an efficient outcome, but no revenue. Note that this low-price 
equilibrium does not require much sophistication. It implements the focal symmetric allocation for this symmetric game. Moreover, even if one player, say $T$, does not understand the equilibrium and thinks of playing straightforwardly, the proposed strategy carries no risk for the other player $M$ because he still obtains the same allocation. Moreover, the fact that $M$ reduces bidding rights from $2 n$ to $n$ in the first round is, of course, a strong signal for $T$ to accept the equal sharing.

\section{Uniqueness of Perfect Equilibrium}

To settle the question of uniqueness of equilibria, we study a simplified version of the GSM auction, which is the "ascending price English clock auction". There, the auctioneer employs a price "clock" that goes up in each round of bidding by one increment, starting from the minimum bid. At each reading of the "clock" bidders are asked to state their demand. When there is no excess demand, the auction ends, and bidders pay the current price for each unit demanded; otherwise, the clock moves up by one increment, and the bidding procedure is repeated, until excess demand has vanished.

Specifically, in every round $t=0,1,2, \ldots, S$, bidders simultaneously submit a bid $B_{i} \in\{0,1,2, \ldots, 2 n\}, i=M, T$, which states how many units they demand at the given price $\Delta t$. Bid sequences must be decreasing over time (to reflect the given activity rule). If $B_{M}(t)+B_{T}(t) \leq 2 n$, the game ends in round $t$ and bidders pay $p_{t}:=\Delta t$ for each of the $B_{i}(t)$ objects they get. If there is excess demand in a round $t<S$, i.e. $B_{M}(t)+B_{T}(t)>2 n$, the game continues to the next round. When the sum of bids exceeds $2 n$ in the final round $S$, every bidder gets 1 item for a price of $p(S)$. This assumption reflects the fact that in the actual auction bidders were not allowed to withdraw their high bids. Technically, it ensures that bidders have an incentive to reduce demand to zero in the last round (see Lemma 1 below).

Outcomes of the auction are denoted by $((k, l), t)$, where $(k, l)$ denotes the allocation ( $k$ units go to player $M$ and $l$ units to $L$ ), and $t$ the round in which the game ends. The price paid per unit is $\Delta t$; hence, it is already fixed by $t$. An allocation $(k, l)$ is "feasible" if $k+l \leq 2 n$, and an outcome $((k, l), t)$ is "loss free" if $w_{j}-j \Delta t \geq 0, j \in\{k, l\}$.

As in other dynamic games, the concept of a Nash equilibrium is too weak, because all relevant outcomes can be supported as an equilibrium 
by using (noncredible) threats.

PROPOSITION 1 (MULTIPLICITY OF NASH EQUILIBRIUM) All outcomes that are loss free and exhibit a feasible allocation are Nash equilibrium outcomes.

ProOF The following strategies are a Nash equilibrium that implements any feasible and loss free outcome $((k, l), t): \forall s \neq t, B_{i}(s)=2 n, i=$ $M, T$, and at round $t, B_{M}(t)=k, B_{T}(t)=l$, provided one has sufficient bidding rights, and otherwise maintain previous bids.

However, the strategies that were invoked in the proof of Proposition 1 are not credible, since they involve the threat to burn all surplus in the event when that equilibrium outcome is not reached. This suggests that applying standard equilibrium refinements should reduce the multiplicity of equilibria.

We can show that, if the price increment $\Delta$ is sufficiently small and there are sufficiently many rounds (as made precise in the assumption below), subgame perfection allows us to identify the competitive allocation $(n, n)$ as the unique SPE allocation, and to show that in a SPE the game ends either in round 0 or 1 . Given this result, it is easy to show that the unique (trembling-hand) perfect equilibrium outcome is $((n, n), 0)$. Therefore, the low price equilibrium, introduced in section 4 , is the unique perfect equilibrium.

AssUMPTION 1 The price increment is small: 1) for all $k=1, \ldots, 2 n-1$ : $2 \Delta<v(k)-v(k+1)$, and 2) $n \Delta<v(n)-v(n+1)$; moreover, 3) the game has sufficiently many rounds: $\Delta S>v(1)$.

Part 1) of Assumption 1 says that the price clock "ticks" at least twice in between two neighboring marginal valuations. Part 2) says that bidders prefer obtaining $n$ units at a price equal to one increment above the competitive equilibrium price, $v(n+1)+\Delta$, to obtaining $n-1$ units at price $v(n+1)$. Part 3 ) says that if the game is ever played until the very last round, $S$, the price clock must already have reached a level above the highest marginal valuation.

THEOREM 2 (UNIQUENESS) In every subgame perfect equilibrium, both bidders get $n$ objects, and the game ends either in round 0 (low price equilibrium) or in round 1 . The corresponding price is equal to zero, resp. equal to $\Delta$. 
The unique trembling-hand perfect equilibrium is the low price equilibrium which ends in round 0 at price zero.

The proof of the Theorem is decomposed into the the following sequence of Lemmas. It uses backward induction. Therefore, the proof starts with the final round.

LEMMA 1 In the final round $S, B_{i}(S)=0$ is the strictly dominant strategy.

Proof If a player bids $B_{i}(S)>0$ he gets one or more items at a price that exceeds $v(1)$; this is strictly worse than bidding $B_{i}(S)=0$, regardless of what the other player does.

Having shown that players reduce demand to zero in round $S$, it is easy to show that this is also the case for all preceding rounds in which the price exceeds the highest marginal valuation $v(1)$.

LEMMA 2 Every subgame perfect equilibrium of a subgame that starts in $t \geq T_{1}$ assigns zero objects to both players.

The unique trembling-hand perfect equilibrium prescribes to bid zero in every round $s \geq t$.

Proof The proof is by backward induction. We know already that in the final round, both players bid zero. So suppose that for every subgame starting in $s>t$, the payoff for both players is zero.

In the reduced game at round $t, B_{i}(t)=0$ is the weakly dominant strategy because for positive bids the game either continues with the same payoff zero or one gets some items at a price which exceeds the valuation. Hence, $B_{i}(t)=0, i=M, T$ is the unique trembling-hand perfect play in round $t$.

There are other SPE in which the game continues for a while which lead to the same allocation $(0,0)$.

We now go a step further and consider the rounds between $T_{2}$ and $T_{1}-1$, where the price is above $v(2)$ and below $v(1)$. To start with, we consider the round just before the valuation $v(1)$ is crossed.

LEMMA 3 Consider subgames starting in round $t=T_{1}-1$ : 
1. If one bidder has less than $2 n$ bidding rights, the unique SPE play is $B_{i}(t)=1$; the resulting allocation is thus $(1,1)$.

2. If both bidders still have $2 n$ bidding rights, the unique tremblinghand perfect play is $B_{i}(t)=1$; there are other SPE in which both players play $B_{i}(t)=2 n$ in round $t$ and the resulting allocation is $(0,0)$.

Proof We have $v(1)-p_{t}>0>w_{k}-k p_{t}, k=2,3, \ldots, 2 n$. Thus, $B_{i}(t)=1$ is the weakly dominant strategy and the unique best reply to every bid $B_{j}(t)<2 n,(j \neq i)$. This proves the first part.

Given that the other player plays $B_{j}(t)=2 n$, player $i$ is indifferent between all bids. Thus, $B_{M}(t)=B_{T}(t)=2 n$ is also part of an equilibrium, which, however, is not trembling-hand perfect because it uses weakly dominated strategies.

Hence, at time $T_{1}-1$ there can be "bad" equilibria in which the auction continues although this is against the interest of both players. However, these bad equilibria can be avoided in earlier rounds by reducing bidding rights.

LEMMA 4 Every subgame perfect equilibrium of a subgame which starts in $t=T_{1}-2$ assigns one object to the players, and the game ends either in round $T_{1}-2$ or $T_{1}-1$.

ProOF If one player has less than $2 n$ bidding rights, this is as in the preceding Lemma. The only problem is when both players have $2 n$ bidding rights and plan to bid $B_{i}\left(T_{1}-1\right)=2 n$ in round $T_{1}-1$ given that they have played $B_{i}\left(T_{1}-2\right)=2 n$ in round $T_{1}-2$. But in this case, $B_{M}\left(T_{1}-2\right)=2 n$ is no longer a best reply to $B_{T}\left(T_{1}-2\right)=2 n-$ by bidding $B_{M}\left(T_{1}-2\right)=1$ player $M$ ensures a strictly positive payoff in round $T_{1}-1$, whereas he gets a zero payoff from bidding $B_{M}\left(T_{1}-2\right)=2 n$.

LEMMA 5 Every subgame perfect equilibrium of a subgame which starts in $T_{2} \leq t \leq T_{1}-2$ assigns one object to the players and ends in round $t$ or $t+1$.

Proof As in the preceding Lemma. 
Therefore, in round $T_{2}$, when prices have crossed $v$ (2) and the demand of both players is 1 , the unique subgame perfect allocation is $(1,1)$, and the game stops either immediately or after one round. Essentially the same reasoning applies to all rounds after $T_{n+1}$, where aggregate demand is less than $2 n$.

LEMma 6 Consider subgames starting in round $t=T_{k}-1, k=2, \ldots, n$ :

1. If one bidder has less than $2 n+1-k$ bidding rights, the unique SPE play is $B_{i}(t)=k$; the resulting allocation is thus $(k, k)$.

2. If both bidders still have more than $2 n+1-k$ bidding rights, the unique trembling-hand perfect play is $B_{i}(t)=k$; there are other SPE in which the game ends in round $T_{k}$ with the allocation $(k-1, k-1)$.

Proof In the first case, $B_{i}(t)=k$ is the strictly dominant strategy.

In the second case, $B_{i}(t)=k$ is still the best reply to all bids $B_{j}(t) \in$ $\{0, \ldots, 2 n-k\}$.

For $B_{j}(t)=l>2 n-k$, two cases are possible.

1. The best reply for $i$ is $B_{i}(t)=2 n-l$, but then $(2 n-l, l)$ is not part of an equilibrium because the best reply to $B_{i}(t)=2 n-l$ is $B_{j}(t)=k$.

2. Or the best reply is $B_{i}(t) \in\{k-1, \ldots, 2 n\}$. Then there are additional SPE plays which lead to an allocation $(k-1, k-1)$ and the game ends in round $T_{k}$ (compare the proof of Lemma 3 ).

LEMMA 7 Every subgame perfect equilibrium of a subgame which starts in $T_{k+1} \leq t \leq T_{k}-2, k=2, \ldots, n$ assigns $k$ object to both players and the game ends in round $t$ or $t+1$. If one player has less than $2 n+1-k$ bidding rights, the game ends in round $t$.

ProOF See Lemma 4.

We know from Lemma 7 that after prices have crossed the "competitive equilibrium price", $v(n+1)$, both players get $n$ objects. Moreover, every player can force the game to stop in round $T_{n+1}$ by reducing bidding rights to $n$. The important step is now to show that this extends to all previous rounds. Bidders anticipate correctly that the allocation will be $(n, n)$ and therefore reduce demand to $n$ right from the beginning. 
LEMMA 8 Every subgame perfect equilibrium of a subgame which starts in $t=T_{n+1}-1$ assigns $n$ objects to both players and the game ends in round $T_{n+1}-1$ or $T_{n+1}$.

ProOF The unique best reply to $B_{T}(t)=n$ is $B_{M}(t)=n$. Hence, $(n, n)$ is a SPE play.

Now consider the bid $B_{T}(t)=n+k$ for $k=1, \ldots, n$. We show that it is not a best response for $M$ to accept the allocation $(n-k, n+k)$. The payoff from accepting is $w_{n-k}-(n-k) p_{t}$, whereas the payoff from playing $B_{T}(t)=n$ is $w_{n}-n p_{t+1}$. Since $p_{t} \leq v(n+1)$, we have

$$
\begin{aligned}
w_{n}-n p_{t+1}-\left(w_{n-k}-(n-k) p_{t}\right)= & v(n)+v(n-1)+\ldots \\
& +v(n-k+1)-k p_{t}-n \Delta \\
\geq & k(v(n)-v(n+1))-n \Delta .
\end{aligned}
$$

By Assumption 1, this term is positive. Hence, $B_{T}(t)=n$ is a strictly better reply than $B_{T}(t)=n-k$. We conclude that the allocation is $(n, n)$ if the game ends in round $t$.

Given that $T$ plays $B_{T}(t)=n+k, B_{M}(t)=n$ need not be the unique best reply. Therefore, the game can continue to round $t+1$. But it cannot continue beyond $t+1$ because by Lemma 7 a player can force the game to stop in round $t+1$ by reducing bidding rights to $n$.

LEMMA 9 Consider a subgame starting in $t<T_{n+1}$. Then every SPE of the subgame starting in $t$ ends in $t$ or $t+1$ with the allocation $(n, n)$.

PROOF By backward induction from the preceding Lemma.

Applying the last Lemma to the first round $t=0$, we see that the game ends with the outcome $((n, n), 0)$ or $((n, n), 1)$. This finishes the first part of the proof of Theorem 2.

Given that the equilibrium allocation is always $(n, n)$, it is of course weakly dominant for both players to bid $n$ in round 0 . Hence, the unique trembling-hand perfect equilibrium play leads to a symmetric partition $(n, n)$ at zero prices. This proves the second part of the Theorem.

We close the analysis with an intuitive remark on the role of the activity rule in explaining the early end of the auction. For example, consider a Nash equilibrium in which $T$ obtains $n+1$ and $M n-1$ units at price 0 , 
because $T$ threatens $M$, along the following lines: "if you do not concede and reduce demand to $n-1$ units, I will keep raising the price until $n$ units are less attractive than obtaining $n-1$ at price 0 ." Then, the activity rule prevents that this can be part of a subgame perfect equilibrium, for the following reason. Consider the subgame where $M$ maintains $n$ (rather than $n-1$ ) bidding rights at price zero. Then, the activity rule entails that $T$ can stop the auction in the following round by reducing his demand to $n$ units. Whereas if $T$ executes his above threat, in the end he also obtains no more than $n$ units, however at higher prices. Therefore, concession yields a higher payoff than executing the threat. This illustrates how the activity rule destroys the credibility of the threat strategies that support asymmetric allocations.

\section{Conclusions}

The present paper has not only shown that low price outcomes may be an equilibrium in a simultaneous ascending-bid auction, which is the typical format of spectrum auctions. We showed that the low price equilibrium that implements the efficient allocation is actually a perfect equilibrium, and indeed the unique perfect equilibrium of that game. This lends support for predicting a low price outcome, which indeed occurred in the German GSM auction, without stipulating any sort of open or tacit collusion among bidders. The strength of this prediction is that it uniquely explains a seemingly collusive outcome from a strictly noncooperative perspective.

Several observers of spectrum auctions in the US, such as WEBER [1997], have already observed that, in a simultaneous ascending-bid auction, bidders may find it in their interest to strategically reduce aggregate demand while prices are still low, relative to their valuations, and actually seem to engage in such bidding practices. In this perspective, the GSM auction in Germany is just a particularly strong and clear cut example, which is not blurred by other issues, like the coordination problems due to the auctioning of concrete (rather than abstract) and regionally restricted frequencies, that plagued the spectrum auctions in the US. Apart from documenting this extreme example of demand reduction, the main value added of the present paper is that it provides a strong noncooperative equilibrium foundation for the observed pattern of strategic demand reduction behavior. 
The main limitation of the present paper is that we assumed bidders are completely informed about each others' valuations. While this was an appropriate assumption in the case of the GSM auction in Germany, it may be a limitation when it comes to apply it to other another context.

\section{References}

AUSUBEL, L. AND J. SCHWARTZ [1999], "The ascending auction paradox,” Working paper, University of Maryland.

BACK, K. AND J. ZENDER [1993], “Auctions of divisible goods," Review of Financial Studies, 6, 733-764.

CRAmton, P. AND J. A. Schwartz [2000], "Collusive bidding: lessons from the FCC spectrum auctions," Journal of Regulatory Economics, $17,229-252$.

ENGELBRECHT-WIGGANS, R. AND C. KAHN [1998], "Low revenue equilibria in simultaneous auctions," Working paper, University of Illinois.

KLEMPERER, P. [2000], “What really matters in auction design,” Working paper, Nuffield College, Oxford University.

MCAFEE, R. AND J. MCMiLlAN [1996], "Analyzing the airwaves auction," Journal of Economic Perspectives, 10, 159-176.

Menezes, F. M. [1996], "Multiple-unit English auctions," European Journal of Political Economy, 12, 671-684.

Milgrom, P. [1998], "Game theory and the spectrum auctions," European Economic Review, 42, 771-778.

Rubinstein, A. [1982], "Perfect equilibrium in a bargaining game,” Econometrica, 50, 97-109.

WEBER, R. J. [1997], "Making more from less: strategic demand reduction in the FCC spectrum auctions," Journal of Economics and Management Strategy, 6, 529-548.

WILSON, R. [1979], “Auctions of shares," Quarterly Journal of Economics, 93, 675-689. 